\title{
La supervision indirecte différée (SID), une méthode pédagogique adaptée aux stages en médecine générale du troisième cycle
}

M onsieur le rédacteur en chef,

La mise en autonomie du résident en médecine générale nécessite la construction d'outils d'évaluation formative adaptés. A G renoble, nous avons mis en place un outil appelésupervis son indirecte différée(SID). Nousaimerions par cette lettre rapporter l'intérêt de cette expérience.

En France, letroi sèmecycledesétudesen médecinegénérale est d'une durée de trois années pour les rési dents (étudiant en formation en médeane générale) qui ont commencéleur cursus en novembre 2001 (il était auparavant de ainq semestres dont un stage en médecine générale). Un tiers du temps de formation totale peut se dérouler dans des structures demédecinegénéraleavec unephased'autonomie de plus en plus importante. Dèlemoisdenovembre2003, les résidents pourront réaliser un deuxième stage en méde cine générale.

La structuredescabinetsmédicaux demédecine généraleett majoritairement composée de cabinets li béraux (médecin en solo ou en groupe). Les structures intégrées regroupant différents corps de métier de la santé sont exceptionnelles, défavorisées par les statuts professionnels, avec des plateaux techniquessouvent modestes.

Le mode d'exercice libéral, le libre choix par le patient de son médecin, ainsi que le paiement à l'acte des médecins renforcent une relation singulière médean-patient.

La place du résident dans ce binôme est préparée, æe fait progressivement d'une phase d'observation, dite passive, à une phased'autonomie, dite active. Lors dela phase active, il effectue seul des actes médicaux pour les patients de ses maîtres destage, avec la possibilitéde leur faire appel en cas de besoin. Cette disponibilité « instantanée » permet de répondre aux problèmes ponctuds, mais ne permet pas une analyse comportementale du résident.

Cette analyse nécessite une supervision indirecte, le maître de stage n'assistant pas directement à la consultation. L'autonomie du résident s'accompagne d'une évaluation que nous avons choise formative, la supervision indirecte différée (SID ).

\section{Intérêt d'une supervision indirecte différée}

Lesétudiants sont, tout au long de leur cursus du deuxième et troisième cycle, entraînés aux méthodes d'apprentissage gl obal centrésur le malade, avec l'apprenti ssage au rai sonnement clinique, l'apprentissage par problème et les évaluations correspondantes

D ans les stages de médecine générale, ils sont progressivement mis en situation d'autonomie. Après une phase d'observation dite passive puisune phase semi-active où les actes médicaux sefont sousla supervision di recte du maître de stage, commence la phase d'autonomie progressive avec une à deux consultations en solo, puis une demi-journée, une journéeet enfin plusieursjours.

Cettegradation permet au rés dent de prendreen comptele suivi au long cours et les consultations itératives ou programmées d'un même patient, de développer la notion de prise en charge globale. Cette mise en situation nécessite une évaluation ${ }^{1-3}$.

Au sein de la faculté de médecine de Grenoble, nous avons dès la mise en place du premier semestre de médecine générale en 1997, privilégié l'autonomie, en autorisant la possibilité pour le réident d'effectuer 540 actes médicaux sans supervis on directe, lors du premier sage de six mois $C^{\prime}$ 'et cette liberté qui nous a amenés progressivement à la miseen placedela supervision indirectedifférée. NousvouIonsfai rebénéficier de notre expériencelesfacultésqui n'ont pas encore misen place cette large autonomie.

Au cours du deuxièmestage en médecine générale, l'activité du rés dent au cabinet nesefera qu'en autonomie. 


\section{Lettre à l'éditeur}

\section{Préparation de la SID}

Durant la phase d'observation, il est demandé au réident qui assiste aux consultations de noter tous les éléments qui lui posent problème, quels qu'ils soient. II fait alors ce que nous avons appeléle recueil d'événements critiques $A$ la fin des consultations, hors la présence du patient, toutes ces quetions sont posées au maître de stage qui jusifi eses attitudes et décisions. Cette phase est primordiale, car l'étudiant qui a critiquéacceptera de l'êtrelorsdela phased'autonomie.

Toutes lessituationsd'apprentissagenécessitent uneanalyse; dans ce cas, il sagit d'une évaluation formative ne concernant quele résident.

D urant la phase d'autonomie, le résident liste les consultations.

Tous les actes effectués par le résident devraient êtreanalysés En pratique il n'est pas certain que cette exhaustivité soit pertinente et nous avons considéré que le rendement de la SID pouvait être optimisé en analysant certainsactes

C'et par le recuél d'informations du résdent et éventuelle ment des patients qu'elle sera réaliséé de manière différée dans le temps.

La SID et une tentative de réponse à l'analyse et à l'évaluation du comportement de l'étudiant dans des situations complexes, variées et réelles.

\section{Quand et comment réaliser une séance deSID ?}

La SID et réalisée:

- à la demande du résident ou des maîtres de stage, à tout moment en cas de problème important;

- de manière systématique après un certain nombre d'actes effectués par le résident. Au début de l'autonomie, il faut procéder à une analyse rapidement, en fin de journé $s$ possible. A prèsun certain tempsl'analysepeut sefaire plus à distance;

- de manière informelle, au gré de moments privilégiés conjonctures;

- on ne peut pas donner de règle stricte; chaque résident, chaquemaître de stage et chaque unité pédagogi quelocale (I'U PL constituée de deux à trois maîtres de stage pour chaque résident) abordent l'autonomie de façon différente et les besoins de réassurance pour chacun varient d'un semestreà l'autre.
N ous analysons:

- tous les actes où le résident a rencontré un problème, c'età-dire: ceux pour lesquelsil a fait un appel itératif à un de ses maîtres de stage et ceux où il a repéré un problème;

- desactes prisau hasard desconsultations. Cesondagealéatoi re es nécessaire car il peut permettre de mettre en évidence des problèmes comportementaux qui n'ont pas été perçus par le résident ;

- les actes pour lesquels une information extérieure nous permet de penser qu'un problème a pu survenir : informations par les patients, les correspondants spéciali istes, les paramédicaux, les laboratoires d'analyse médicale ou les institutions (cai sses d'assurance maladie, D D ASS, etc.).

Les maîtres de stage doi vent vérifier au fur et à mesure des SID que la plupart des situations courantes en médecine générale sont analysées

\section{Comment réaliser une SID ?}

$N$ otre organisation en UPL avec deux à trois maîtres de stage permet différentes modalités:

1. C'est le maître de stage qui a confié ses patients au résident qui procède à la SID ; c'est lui en effet qui connaît le mi eux ses patients et peut ai der à résoudre le problème.

2. II faut prévoir quelques séances où tous les maîtres de stage sont présents pour la séance de SID ; ces séances permettent à l'étudiant de confronter les anal yses de chacun des maitres de stage sur des situations complexes, de faire appel si besoin aux données de la médecine basée sur les niveaux de preuve et de forger sa propre analyse U ne analyse comportementale par SID est favorisée par un recueil standardisédes données

N ous essayons différentes grilles de recueil et d'exposition de problèmes Cesgrilles doi vent reter soupleset neservent que de canevas proposé au rés dent pour synthéiser son problème En pratique nous utilisons la trame proposée par la SF M G ${ }^{(4)}$ pour l'exposition de cas dans les groupes de pairs, maisleplus souvent lerésdent exposeles stuationsdiniques qui lui ont posép problème de façon spontanée et le maître de stage peut êtreamenéà reformuler.

L'ensemble de l'analyse reste oral, il n'est pas nécessaire sauf exception, de faire appel à un support écrit. 


\section{Lettre à l'éditeur}

A partir dela présentation du réident, le ou les maîtres de stage doivent pouvoir classer le problème en différentes catégories N ous avons arbitrairement classé les problèmes en terme de compétence gestuelle, organisati onnelle (réseau, temps matérie), décisionnelle et relationnelle.

A chaque problème sont apportés une solution ou des éémentsd'orientation. Lessituationsti rées au hasard et qui neposent pas de problème sont quand mêmeanalysés. Les moments où une séance de SID est réali sée sont, dans notre expérience, les visitesà domicile, dans le véhicule du médecin, au coursdesrepasprisen commun, en fin dejournée, lors de séances programmées et à tout moment jugé opportun par lemaître de stage.

L'histoire des patients n'est pas terminée après la séance de SID et il est important qu'après celled et même après le stage, le maître de stage puisse apporter les informations majeures concernant les patients qui ont été «partagés » avec le résident.

\section{Conclusion}

L'autonomie du résident en stagedemédecine générale doit faire l'objet d'une évaluation continue pour dépister les stuations à risque et même les étudiants à problèmes Les conduitesnon conformesaux bonnes pratiques voire dange reuses sont alors repérées et corrigées par le maître de stage. La mise en situation d'autonomie privilégi el'apprenti ssage plutôt que l'enseignement mais ne doit pas exclure un contrôle a posteriori.

Jean-Pierre Jacquet, Patrick Imbert Faculté de médecine de Grenoble, France.

\section{Références}

1. Vidal M, Lauque D, N icodemeR, BrosB, Arlet P. Les outils pédagogiques pour le stage des rési dents au cabinet du médecin généraliste français. Pédagogie M édicale 2002, 3 : 33-7.

2. Jouquan J. L'évaluation des apprentissages des étudiants en formation médicale initiale. Pédagogie M édicale 2002, 3 : 38-52.

3. Turgeon J, St-H ilaire S. La supervision directe en médecine familiale. L'expérience d'une résidente. Pédagogie médicale 2001, 2 : 199-205.

4. Jacot P. Société Française de M édecine G énérale, http//www.sfmg.org, Groupe de pairs, Grille de pré sentation de cas cliniques.

Correspondance: D r Jean-Pierre Jacquet, immeuble C roisée Bellevue F-73230 Saint Jean D 'Arvey Courriel : jp.jacquet@medsyn.fr

\section{www,pedagogle-medicale.org \\ un site à votre disposition}

Votre revue est disponible sur Internet. Vous y retrouverez les informations générales sur la revue, les adresses de contact, les sommai res des différents numéros parus, les directives aux auteurs etc. Les éditoriaux et toutes les rubriques de la section «V ie pédagogique »y sont disponibles « in extenso » en format PDF (lisibles avec Acrobat Reader). Au fur et à mesure de leur publication, les fiches pratiques seront misesen ligne sur le site Vous pourrez ainsi les télécharger, les imprimer chez vous, et vous construire progressivement votre propre manuel pratique. D ivers liens vers les auteurs des articles déjà publiés et les organisations du comité de parrainage sont également disponibles.

Un moteur de recherche spécifique au site vous permet désomais de rechercher des articles ou résumés par mots-clés, auteur, etc. N'hésitez pas à transmettre vos opinions sur les articles (via le forum de discussion) ou directement à la rédaction. Les commentaires les plus intéréssants seront publiés dans les pages de la revue. Enfin, nous attendons vos réactions à l'enquête de la revue, publiée dans le numéro 4.1 et qui est accessible directement sur le site à partir de la page d'accueil.

Le site www.pedagogie-medicale.org se veut un lien amical et fonctionnel entre tous ceux qui sont impliqués dans la formation médicale. Le site doit leur permettre de partager leurs préoccupations et leurs solutions dans leur action pour la formation initiale et la formation continue de nos professionnels de santé.

La rédaction 